University of South Florida

DIGITAL COMMONS

Digital Commons @ University of

@ UNIVERSITY OF SOUTH FLORIDA

South Florida

Mental Health Law \& Policy Faculty

Publications

Mental Health Law \& Policy

2016

\title{
Prevalence and Predictors of Substance-Related Emergency Psychiatry Admissions
}

\author{
M. Scott Young \\ University of South Florida, syoung1@usf.edu \\ Kathleen A. Moore \\ University of South Florida, kamoore@usf.edu
}

Follow this and additional works at: https://digitalcommons.usf.edu/mhlp_facpub

Part of the Health Law and Policy Commons, and the Mental Disorders Commons

\section{Scholar Commons Citation}

Young, M. Scott and Moore, Kathleen A., "Prevalence and Predictors of Substance-Related Emergency Psychiatry Admissions" (2016). Mental Health Law \& Policy Faculty Publications. 827.

https://digitalcommons.usf.edu/mhlp_facpub/827

This Article is brought to you for free and open access by the Mental Health Law \& Policy at Digital Commons @ University of South Florida. It has been accepted for inclusion in Mental Health Law \& Policy Faculty Publications by an authorized administrator of Digital Commons @ University of South Florida. For more information, please contact digitalcommons@usf.edu. 


\section{Prevalence and Predictors of Substance- Related Emergency Psychiatry Admissions}

\section{Abstract}

Background: Individuals commonly present for emergency psychiatry services for reasons related to their use of alcohol or illicit drugs. This study assessed the prevalence of these phenomena and explored characteristics distinguishing emergency psychiatry admissions with versus without presenting problems related to substance use.

Methods: Data included standardized emergency psychiatry intake interviews from 2,161 consecutive admissions to three hospital-based emergency psychiatry departments in Florida's Tampa Bay area. Admissions were classified as substanceinvolved if substance use was ascertained to be related to the presenting problem(s). Cases with only substance-related presenting problems were classified as substance-only admissions. Descriptive statistics compared substance-involved admissions to those whose presenting problems were not related to substance use. A logistic regression determined the characteristics most predictive of substance-involved admissions; similarly, a second logistic regression analysis was used to predict substance-only admissions.

Findings: A substantial number of emergency psychiatry admissions ( $n=507 ; 23.5 \%)$ were identified as being substance-involved. These patients were more often male, single, and unemployed as compared to those whose presenting problems were not linked to substance use. Substance involvement was documented as the sole presenting problem for a sizable portion ( $n=171 ; 7.9 \%)$ of the emergency psychiatry department admissions. This model was similar to the previous one except that gender, employment status, and insurance type were no longer significant predictors; additionally, the second model revealed that separated or divorced participants were more than three times as likely $(O R=3.30, p<0.001)$ as those who were single to present for services for only substance-related reasons.

Conclusion: Though high costs prohibit universal implementation of biologicallybased substance use screening procedures in emergency psychiatry settings, several characteristics have consistently been shown to significantly relate to substance-involved admissions. These characteristics can be quickly and cheaply obtained during a brief interview to trigger a more thorough assessment of the patients' substance involvement, a brief intervention in the emergency department if possible, and/or appropriate referrals for addiction and integrated Co-occurring disorders treatment service.

Keywords: Co-occurring disorders; Emergency psychiatry; Mental health; Addiction; Substance use

\section{Scott Young and Kathleen A Moore}

Department of Mental Health Law and Policy, Louis de la Parte Florida Mental Health Institute, University of South Florida, USA

Corresponding author: M Scott Young

\section{syoung1@usf.edu}

Department of Mental Health Law and Policy, Louis de la Parte Florida Mental Health Institute, University of South Florida, USA.

Tel: 813-974-8734

Citation: Young MS, Moore KA. Prevalence and Predictors of Substance-Related Emergency Psychiatry Admissions. Dual Diagn Open Acc. 2016, 1:1. 


\section{Introduction}

\section{Background}

Studies have long documented the high prevalence and adverse impact of comorbid substance use on the course and treatment of persons with psychiatric problems. The National Institute of Mental Health Epidemiological Catchment Area (ECA) study [1] found that persons with a mental illness had more than twice the rate of alcohol abuse, and four times the rate of drug abuse, compared to persons without a mental illness. Both the ECA [2] and the National Comorbidity Survey [3] found that most people in need of substance abuse treatment services did not receive these services. However, among those who do access addiction treatment services, studies suggest that a large proportion reported obtaining them in the general medical sector, particularly in emergency departments [4-6] or specialty emergency psychiatry settings $[7,8]$. Table 1 summarizes several studies' findings regarding the high prevalence of substance abuse among emergency psychiatry patients. Though rates vary across studies, results indicate that substance abuse is involved with $20 \%$ to $50 \%$ of the cases encountered in emergency psychiatry settings. Even among persons who are homeless, substance abuse has been found to significantly increase the chances of accessing emergency psychiatry services [9]. Unfortunately, emergency medical settings are unlikely to screen for potential substance abuse problems, substantially reducing their patients' chances of receiving substance abuse treatment services.

Demographic and clinical characteristics have been linked to increased risk for substance abuse among emergency psychiatry patients. With respect to demographics, African American ethnicity and male gender have been related to increased risk $[10,11]$. Clinically, studies have documented higher rates of substance use among emergency psychiatry patients with a psychotic disorder [12,13].

Substance abuse presents significant challenges to providing emergency psychiatry patients with the most effective care. First, these patients are more difficult to treat. Compared to emergency psychiatry patients who do not abuse substances, those with substance abuse problems often require higher levels of behavioral management, spend more time in the psychiatric emergency room, and are more likely to present as suicidal [12]. Second, emergency psychiatry patients with comorbid substance abuse problems are typically more costly to treat; this is because they often utilize significantly more emergency department services, including a greater number of emergency department readmissions, as compared to those with no co-occurring substance use issues $[13,14]$. Third, emergency psychiatry patients with substance-related problems are also significantly less likely than those without substance use problems to follow up on referrals made to community treatment agencies [15]. For all of these reasons, it is important to identify and address substance misuse in order to provide optimal care for individuals receiving emergency psychiatry services.

\section{Goals of This Investigation}

The purpose of this exploratory study was to determine the extent to which involvement with alcohol or drugs (i.e., substance involvement) was related to reasons for seeking care in emergency psychiatry settings. Additionally, this study further sought to identify characteristics (i.e., demographic, medical, clinical, behavioral, and insurance-related) that distinguish emergency psychiatry patients whose presenting problem(s) relate to their substance use, and also to distinguish admissions whose only presenting problem(s) were related to substance use.

\section{Methods}

\section{Study design}

This study utilized interview methods with all consecutive patients admitted to one of three participating emergency psychiatry departments over a three month period.

\section{Setting}

Data collection occurred at one of three participating hospitals in Florida's Tampa Bay area. Data entry and analysis took place at University of South Florida.

\section{Selection of participants}

The sample included 2,161 consecutive patient admissions to one of three participating emergency psychiatry service departments. Participants were rather evenly distributed across the three hospitals, with each hospital contributing 662, 720, and 779 cases. Participants arrived at a hospital's emergency psychiatry service department by one of two methods. In the more common scenario, participants $(n=1,238)$ were triaged to emergency psychiatry services after an emergency room intake nurse determined the patient to have a psychiatric history or active symptoms of a psychiatric disorder. Alternatively, other participants $(n=923)$ presented directly to emergency psychiatry department because they were brought to the hospital under Florida's mental health-related involuntary commitment law, more commonly known as the Baker Act $[16,17]$. Because all three participating hospitals were designated as "Baker Act receiving facilities," they were allowed to receive patients in this manner.

\section{Methods of measurement}

Hospital intake staff completed intake interviews on all patients presenting for emergency psychiatry services at one of the three participating sites. Interviews were guided by an intake assessment form that was standardized across all three participating hospitals. Interview items concerned participants' demographics, arrival mode, referral source, legal status, medical history, and insurance coverage. Information on each patient's presenting problem(s) was documented in narrative text format. Interviewers wrote information directly onto the standardized intake assessment form, and the information was later entered into a computer database. Interviewers used interview findings as well as their professional judgment when completing the presenting problem(s) field.

\section{Data collection and processing}

Before any data were collected, all study procedures were first 
Table 1 Rates of substance-involvement among emergency psychiatry patients.

\begin{tabular}{|c|c|c|c|c|}
\hline Study & Sample & Screening Method & Sample Size & $\begin{array}{l}\% \text { with Substance } \\
\text { Involvement }\end{array}$ \\
\hline Atkinson [31] & Psychiatric ER patients & Psychiatric Interview & 503 & $53.7 \%$ \\
\hline Fong et al., [8] & Psychiatric ER patients & Psychiatric Interview & 3,150 & $40.5 \%$ \\
\hline Solomon and Gordon [15] & Psychiatric ER patients & Psychiatric Interview & 114 & $39.0 \%$ \\
\hline $\begin{array}{l}\text { Szuster, Schanbacher, and } \\
\text { McCann, [38] }\end{array}$ & Psychiatric ER patients & Psychiatric Interview & 343 & $33.2 \%$ \\
\hline Lejoyeux et al., [35] & Psychiatric ER patients & Psychiatric Interview & 104 & $37.5 \%$ alcohol dependent \\
\hline McNeil and Binder, [26] & Psychiatric ER patients & Psychiatric Interview & 2,784 & $35.1 \%$ substance use \\
\hline Lieberman and Baker, [36] & $\begin{array}{c}\text { Psychiatric ER patients later } \\
\text { admitted to inpatient } \mathrm{MH} \\
\text { unit }\end{array}$ & Psychiatric Interview & 50 & $\begin{array}{c}20.0 \% \text { alcohol abuse, } 16.0 \% \\
\text { nonalcoholic substance } \\
\text { abuse }\end{array}$ \\
\hline $\begin{array}{c}\text { Schiller, Shumway, and } \\
\text { Batki, [10] }\end{array}$ & Psychiatric ER patients & UTS & 122 & $43.4 \%$ \\
\hline Elangovan et al. [33] & Psychiatric ER patients & UTS & 218 & $34.4 \%$ \\
\hline $\begin{array}{l}\text { Dhossche and Rubinstein, } \\
\text { [28] }\end{array}$ & Psychiatric ER patients & UTS & 1,526 & $27.7 \%$ \\
\hline Sanguineti and Samuel, [39] & $\begin{array}{c}\text { Involuntary psychiatric ER } \\
\text { patients }\end{array}$ & UTS & 129 & $38.8 \%$ \\
\hline Sanguinetti and Brooks, [27] & $\begin{array}{l}\text { Involuntary psychiatric ER } \\
\text { patients }\end{array}$ & UTS & 247 & $34.0 \%$ \\
\hline $\begin{array}{c}\text { Claassen, Gilfillan, Orsulak, } \\
\text { Carmody, Battaglia and } \\
\text { Rush, [32] }\end{array}$ & $\begin{array}{l}\text { Psychotic patients seeking } \\
\text { services in an urban } \\
\text { psychiatric ER }\end{array}$ & UTS & 112 & $21.4 \%$ \\
\hline $\begin{array}{c}\text { Gilfillan, Claasen, Orsulak, et } \\
\text { al., [32] }\end{array}$ & $\begin{array}{l}\text { Nonpsychotic Psychiatric ER } \\
\text { patients without a primary } \\
\text { substance abuse diagnosis }\end{array}$ & UTS & 93 & $34.4 \%$ \\
\hline $\begin{array}{l}\text { Wildt, Andreis, Auffahrt, } \\
\text { Tennenborn, Kropp and } \\
\text { Ohlmeier, [14] }\end{array}$ & Psychiatric ER patients & BAC & 2,194 & $39.7 \%$ \\
\hline $\begin{array}{l}\text { Breslow, Klinger, and } \\
\text { Erickson, [12] }\end{array}$ & Psychiatric ER patients & BAC and UTS & 294 & $32.0 \%$ \\
\hline Strakowski et al., [37] & Psychiatric ER patients & $\begin{array}{c}\text { Psychiatric interview and } \\
\text { UTS }\end{array}$ & 490 & $33.5 \%$ \\
\hline
\end{tabular}

Notes: UTS $=$ urine toxicology drug screen. $\mathrm{BAC}=$ blood alcohol concentration test.

approved by the University of South Florida's Institutional Review Board, including appropriate methods related to informed consent. Upon arrival at the emergency psychiatry service department, all participants completed the standardized intake assessment interview with an emergency psychiatry department nurse who was employed by the hospitals and had no knowledge of this study's focus on substance involvement.

\section{Outcome measures}

The intake survey included an open-ended question for describing the presenting problem(s) and reason(s) for seeking emergency psychiatry services. This item was coded to indicate which of the following eight problems contributed to each patient's immediate need for emergency psychiatry services: 1) violence, 2) suicide, 3) police involvement, 4) domestic violence, 5) involvement with alcohol and / or drugs (substance involvement), 6) medication issues, 7) other mental health problems (e.g., panic attack, hearing voices), and 8) other non-psychiatric problems. Problem categories were not mutually exclusive, so multiple presenting problems could be related to a patient's need for emergency psychiatry services. Presenting problems related to alcohol and drugs were labeled "substance-involved" and generally included cases in which patients were intoxicated or withdrawing from substances, suffered injuries as a result of substance use, or were experiencing medical complications of drug use. A second variable called "substance-only" admissions was created to indicate cases whose only presenting problem was listed as substance involvement.

Importantly, police involvement was not classified as a presenting problem if the police were listed as the arrival mode but were not mentioned in the presenting problem text field. The "other nonpsychiatric problem" category was used to document instances in which additional non-psychiatric problems accompanied the psychiatric emergency, and no patients presented with only an "other non-psychiatric problem."

\section{Primary data analysis}

All analyses were performed using SPSS 22.0. In the first phase of the analyses, demographic, clinical, and medical information was compared between cases with versus without substance involvement documented among the presenting problems. Chi-square tests were used to compare the two groups on 
categorical variables, and $t$-tests were used for comparisons involving continuous variables. Levene's test of homogeneity of variances was performed prior to each $t$-test. If the assumption of homogeneity of variances was violated (i.e., variances were found to be significantly different at $p<0.1)$, then a robust $t$-test was performed instead [18].

The second phase of the analyses involved a multivariate binary logistic regression to determine the extent to which various factors distinguished emergency psychiatry cases with substanceinvolved presenting problems. All significant variables from the first phase of the analyses were simultaneously entered as predictors, with the dependent variable being a dichotomous variable indicating presence or absence of substance involvement among the presenting problems. The predictors were simultaneously entered in a forward step-wise fashion to assess their ability to predict substance-involved emergency psychiatry admissions while controlling for the effects of other variables in the model. A second logistic regression model was run in a similar manner using the same set of predictors, but this equation was set to predict admissions whose only presenting problem was listed as substance involvement. While the first regression equation predicted whether participants' presenting problems were substance-related, the second sought to find predictors associated with admissions whose presenting problems were documented as attributable solely to substance involvement.

\section{Results}

\section{Characteristics of study subjects}

Table 2 presents the overall sample demographics in the rightmost column. Patients averaged just under forty years of age $(M=39.2, S D=17.1)$ with a slight majority being female (51.9\%). Most patients were single $(61.3 \%)$, followed by those who were separated / divorced (18.7\%), married (15.8\%), or widowed (4.3\%). Patients most commonly described their living arrangements as living independently (29.7\%), with a spouse or relatives $(26.5 \%)$, or with parents $(20.6 \%)$. Most patients reported being either on disability $(36.0 \%)$ or unemployed $(25.5 \%)$, with fewer reporting being employed (19.0\%) or a student $(12.2 \%)$. Patients were referred to the hospital from a variety of sources, though they were most commonly referred from their residence (56.5\%). Other common referral sources included residential homes (10.6\%) and physicians or hospitals (9.3\%). Patients were most commonly brought to the emergency psychiatry room via law enforcement $(29.7 \%)$, an ambulance $(25.8 \%)$, or family members (18.2\%), though some patients brought themselves to the emergency psychiatry service $(17.3 \%)$. The vast majority of patients were ambulatory (95.2\%) upon presenting for services, with few using walkers $(2.3 \%)$ or wheelchairs $(1.9 \%)$ or being bed bound $(0.4 \%)$. With regard to legal status, nearly half of the admissions presented to the emergency psychiatry department on a voluntary basis (47.4\%), with $52.6 \%$ arriving through Florida's involuntary commitment law (Baker Act). Few patients reported past property destruction (3.7\%), fire setting $(0.9 \%)$, or animal cruelty $(0.5 \%)$. Nearly one in twenty patients $(4.7 \%)$ reported being the victim of sexual abuse, with slightly more reporting victimization by physical abuse (5.4\%). Patients most commonly reported having private $(43.2 \%)$ or public insurance $(37.1 \%)$, and $19.7 \%$ reported being uninsured. Substance involvement was documented as a presenting problem in 507 (23.5\%) admissions. Substance involvement was documented as the sole presenting problem for a sizable portion $(n=171 ; 7.9 \%)$ of the emergency psychiatry department admissions.

Table 2 also compares demographic data between participants whose admission was related versus unrelated to involvement with alcohol or drugs. Results indicate that substance-related admissions were significantly less likely to be female (39.1\% versus $55.9 \%), \chi^{2}(1,2161)=43.93, p<0.001$. There were also significant differences in marital status, $\chi^{2}(3,1973)=19.17, p<0.001$, and living arrangements, $\chi^{2}(4,1732)=88.29, p<.001$. Substance involved admissions were more likely to be separated or divorced and living independently, whereas cases unrelated to substance use were more likely to be single and living with parents or in a residential facility such as a nursing home, group home, or other assisted living facility. These differences in residential status are not likely attributable age differences, as the groups did not differ significantly with regard to average age $(p=0.648)$. There were also significant employment differences, $\chi^{2}(6,1943)=109.33$, $\mathrm{p}<0.001$, though employment rates were low for both groups. Admissions with presenting problems unrelated to substance involvement were most likely to be on disability (39.4\%) or unemployed $(22.0 \%)$, while substance-involved admissions were most likely to be unemployed (37.1\%) or employed (27.9\%).

Cases varying in substance involvement also differed significantly with respect to their referral source, $\chi^{2}(4,1675)=48.18, p<0.001$. Though the group of substance-related admissions was more likely to be referred to the hospital from a private residence, this was still the most common referral source for both groups. Admissions unrelated to substance involvement were more likely to be referred by a residential home, though this is largely explained by the fact that they were more much likely to be living in one. There were also significant between group differences with regard to insurance coverage, $\chi^{2}(2,1861)=102.48, p<0.001$. Substance-involved admissions were more likely to be uninsured, self-pay clients without private or public health insurance coverage, while admissions whose presenting problems were unrelated to substance involvement were more likely to be on public assistance (i.e., Medicaid or Medicare). A comparably sizable proportion in each group had private insurance coverage.

Several clinical and medical characteristics also distinguished admissions whose presenting problems were related to substances (Table 3). Substance-involved admissions were significantly less likely to involve violence, $\chi^{2}(1,2161)=7.83, p=0.005$, or homicidal intent, $\chi^{2}(1,2161)=9.73, p=0.002$. The groups did not differ with regard to several aspects of their behavior during the interview, including their cooperativeness, impulsivity, eye contact, or level of motor activity. With regard to affect, substanceinvolved admission were significantly less likely to be anxious, $\chi^{2}(1,2161)=4.72, p=0.030$, though they were significantly more likely to be depressed, $\chi^{2}(1,2161)=6.36, p=0.012$. Medical history data indicated that substance-involved admissions were significantly less likely to have diabetes, $\chi^{2}(1,2161)=11.39$, $\mathrm{p}<0.001$. A robust $t$-test indicated that, on average, substance- 
Table 2 Sample demographics, by substance-involvement.

\begin{tabular}{|c|c|c|c|}
\hline \multicolumn{4}{|c|}{ Emergency Psychiatry Admission Type ${ }^{1}$} \\
\hline & Not Substance-Related & Substance-Related & Total Sample \\
\hline & $(n=1654)$ & $(n=507)$ & $(n=2161)$ \\
\hline Age (Mean, SD) & $39.4(18.2)$ & $38.9(12.6)$ & $39.2(17.1)$ \\
\hline Gender: Female*** & $55.9 \%$ & $39.1 \%$ & $51.9 \%$ \\
\hline \multicolumn{4}{|l|}{ Marital Status*** } \\
\hline Married & $15.5 \%$ & $16.5 \%$ & $15.8 \%$ \\
\hline Single & $63.6 \%$ & $53.9 \%$ & $61.3 \%$ \\
\hline Separated / Divorced & $16.7 \%$ & $25.1 \%$ & $18.7 \%$ \\
\hline Widowed & $4.2 \%$ & $4.5 \%$ & $4.3 \%$ \\
\hline \multicolumn{4}{|l|}{ Living Arrangement*** } \\
\hline Independent & $26.1 \%$ & $42.2 \%$ & $29.7 \%$ \\
\hline W/Spouse or Relatives & $26.1 \%$ & $27.8 \%$ & $26.5 \%$ \\
\hline With Parents & $22.5 \%$ & $13.9 \%$ & $20.6 \%$ \\
\hline W/Non-Relatives & $7.7 \%$ & $12.9 \%$ & $8.9 \%$ \\
\hline Residential Home ${ }^{a}$ & $17.5 \%$ & $3.3 \%$ & $14.3 \%$ \\
\hline \multicolumn{4}{|l|}{ Employment Status*** } \\
\hline Employed & $16.2 \%$ & $27.9 \%$ & $19.0 \%$ \\
\hline Student & $14.4 \%$ & $5.0 \%$ & $12.2 \%$ \\
\hline Unemployed & $22.0 \%$ & $37.1 \%$ & $25.5 \%$ \\
\hline Disability & $39.4 \%$ & $24.9 \%$ & $36.0 \%$ \\
\hline Retired & $8.0 \%$ & $5.0 \%$ & $7.3 \%$ \\
\hline \multicolumn{4}{|l|}{ Referral Source*** } \\
\hline Residence & $54.6 \%$ & $62.9 \%$ & $56.5 \%$ \\
\hline Physician / Hospital & $10.0 \%$ & $7.0 \%$ & $9.3 \%$ \\
\hline Psychiatric Facility & $2.7 \%$ & $2.1 \%$ & $2.6 \%$ \\
\hline Residential Home ${ }^{a}$ & $13.2 \%$ & $2.1 \%$ & $10.6 \%$ \\
\hline Other & $19.4 \%$ & $26.0 \%$ & $21.0 \%$ \\
\hline \multicolumn{4}{|l|}{ Arrival Mode } \\
\hline Self & $17.6 \%$ & $16.5 \%$ & $17.3 \%$ \\
\hline Law Enforcement & $29.3 \%$ & $31.3 \%$ & $29.7 \%$ \\
\hline Family & $19.1 \%$ & $15.4 \%$ & $18.2 \%$ \\
\hline Ambulance & $24.9 \%$ & $28.7 \%$ & $25.8 \%$ \\
\hline Other & $9.2 \%$ & $8.1 \%$ & $9.0 \%$ \\
\hline \multicolumn{4}{|l|}{ Ambulation } \\
\hline Ambulatory & $94.6 \%$ & $97.0 \%$ & $95.2 \%$ \\
\hline Walker & $2.4 \%$ & $2.1 \%$ & $2.3 \%$ \\
\hline Wheelchair & $2.4 \%$ & $0.5 \%$ & $1.9 \%$ \\
\hline Bed Bound & $0.4 \%$ & $0.5 \%$ & $0.4 \%$ \\
\hline Other & $0.3 \%$ & $0.0 \%$ & $0.2 \%$ \\
\hline \multicolumn{4}{|l|}{ Legal Status } \\
\hline Involuntary/Baker Act & $52.2 \%$ & $53.7 \%$ & $52.6 \%$ \\
\hline Voluntary & $47.7 \%$ & $46.3 \%$ & $47.4 \%$ \\
\hline \multicolumn{4}{|l|}{ Past Behavior } \\
\hline Property Destruct. & $4.0 \%$ & $2.6 \%$ & $3.7 \%$ \\
\hline Fire Setting & $0.8 \%$ & $1.2 \%$ & $0.9 \%$ \\
\hline Animal Cruelty & $0.4 \%$ & $0.6 \%$ & $0.5 \%$ \\
\hline Sex Offender & $0.3 \%$ & $0.0 \%$ & $0.2 \%$ \\
\hline \multicolumn{4}{|l|}{ Abuse History } \\
\hline Sexually Abused & $5.0 \%$ & $3.9 \%$ & $4.7 \%$ \\
\hline Physically Abused & $5.6 \%$ & $4.9 \%$ & $5.4 \%$ \\
\hline Any Sexual or Physical Abuse & $8.0 \%$ & $6.9 \%$ & $7.7 \%$ \\
\hline Both Sexual and Physical & $2.7 \%$ & $2.1 \%$ & $2.5 \%$ \\
\hline Insurance Type*** & & & \\
\hline
\end{tabular}




$\begin{array}{cccc}\text { Uninsured } & 15.3 \% & 34.4 \% & 19.7 \% \\ \text { Public (Medicaid / Medicare) } & 42.0 \% & 20.8 \% & 37.1 \% \\ \text { Private } & 42.7 \% & 44.8 \% & 43.2 \%\end{array}$

${ }^{*} \mathrm{p}<0.05, * * \mathrm{p}<0.01, * * * \mathrm{p}<0.001$

aResidential homes include nursing homes, group homes, or assisted living facilities.

${ }^{1}$ Admission type was determined from the presenting problem.

Table 3 Clinical and medical characteristics, by substance-involvement.

\begin{tabular}{|c|c|c|c|}
\hline \multicolumn{4}{|c|}{ Emergency Psychiatry Admission Type ${ }^{1}$} \\
\hline & Not Substance-Related & Substance-Related & Total Sample \\
\hline & $(N=1654)$ & $(\mathrm{N}=507)$ & $(n=2161)$ \\
\hline \multicolumn{4}{|l|}{ Presenting Problem(s) } \\
\hline Suicidal Intent & $45.2 \%$ & $41.6 \%$ & $44.4 \%$ \\
\hline Police Involvement & $13.2 \%$ & $16.2 \%$ & $13.9 \%$ \\
\hline Violence** & $9.3 \%$ & $5.3 \%$ & $8.3 \%$ \\
\hline Homicidal Intent** & $4.4 \%$ & $1.4 \%$ & $3.7 \%$ \\
\hline Domestic Violence & $1.5 \%$ & $1.0 \%$ & $1.4 \%$ \\
\hline Medication Issues & $2.5 \%$ & $1.6 \%$ & $2.3 \%$ \\
\hline Other Non-Psychiatric Problem & $0.8 \%$ & $0.4 \%$ & $0.7 \%$ \\
\hline \multicolumn{4}{|l|}{ Current Behavior } \\
\hline Poor Eye Contact & $18.6 \%$ & $20.3 \%$ & $19.0 \%$ \\
\hline Impulsivity & $15.1 \%$ & $12.8 \%$ & $14.5 \%$ \\
\hline Decreased Motor Activity & $14.1 \%$ & $14.2 \%$ & $14.2 \%$ \\
\hline Increased Motor Activity & $12.8 \%$ & $12.8 \%$ & $12.8 \%$ \\
\hline Uncooperative & $11.5 \%$ & $12.4 \%$ & $11.8 \%$ \\
\hline \multicolumn{4}{|l|}{ Affect } \\
\hline Anxious* & $48.3 \%$ & $42.8 \%$ & $47.0 \%$ \\
\hline Depressed* & $47.6 \%$ & $54.0 \%$ & $49.1 \%$ \\
\hline Flat & $22.9 \%$ & $22.3 \%$ & $22.7 \%$ \\
\hline Angry & $14.3 \%$ & $14.8 \%$ & $14.4 \%$ \\
\hline Euphoric & $1.5 \%$ & $1.6 \%$ & $1.5 \%$ \\
\hline Inappropriate & $5.7 \%$ & $3.6 \%$ & $5.2 \%$ \\
\hline \multicolumn{4}{|l|}{ Medical History } \\
\hline Allergies & $45.4 \%$ & $39.8 \%$ & $44.1 \%$ \\
\hline Hypertension & $12.3 \%$ & $10.7 \%$ & $11.9 \%$ \\
\hline Seizures & $7.1 \%$ & $8.5 \%$ & $7.5 \%$ \\
\hline Diabetes** & $5.6 \%$ & $2.0 \%$ & $4.8 \%$ \\
\hline Thyroid Problems & $2.1 \%$ & $2.0 \%$ & $2.1 \%$ \\
\hline Cancer & $0.9 \%$ & $1.2 \%$ & $1.0 \%$ \\
\hline \# Prescribed Meds***a & $3.3(2.9)$ & $2.4(2.4)$ & $3.1(2.8)$ \\
\hline
\end{tabular}

$*_{p}<0.05, * * p<0.01, * * * p<0.001$

aLevene's test for homogeneity of variance indicated unequal variances, so a robust $t$-test was used.

${ }^{1}$ Admission type was determined from the presenting problem.

involved emergency psychiatry cases reported being prescribed significantly fewer medications (2.4 vs. 3.3$), t(759)=6.301$, $\mathrm{p}<0.001$.

Table 4 presents the results of a multivariate logistic regression predicting admissions with presenting problem related to substance involvement. All variables for the previous analyses that significantly distinguished substance-involved admissions were simultaneously entered as predictors. The overall model was statistically significant, $\chi^{2}(24)=146.61, p<0.001$, Nagelkerke
$R^{2}=0.22$. Several variables were significant predictors of substance-involved admissions. With regard to demographics, results indicated male emergency psychiatry patients were 1.85 times more likely than female emergency psychiatry clients to present for services at least in part due to substance-related reasons, $p<0.001$. Separated or divorced participants were more than twice as likely $(\mathrm{OR}=2.04)$ as those who were single to present for services due at least in part for substance-related reasons, $p=0.002$. The odds of presenting for services due to substance involvement were significantly reduced for patients 
Table 4 Binary logistic regression analysis predicting substance-involved admission.

\begin{tabular}{|c|c|c|c|c|c|}
\hline Predictor Variable & $B$ & $\begin{array}{l}\text { Odds } \\
\text { Ratio }\end{array}$ & $\begin{array}{c}95 \% \text { Wald } \\
\text { Confidence Limits }\end{array}$ & Wald $\chi^{2}$ & $p$ Value \\
\hline Constant & -0.78 & 0.46 & & 6.20 & 0.013 \\
\hline \multicolumn{6}{|l|}{ Demographics } \\
\hline Gender: Male vs. Female & 0.62 & 1.85 & $(1.31-2.62)$ & 12.12 & $<0.001$ \\
\hline Marital Status & & & & 10.74 & 0.013 \\
\hline Married vs. Single & 0.20 & 1.22 & $(0.68-2.16)$ & 0.45 & 0.505 \\
\hline Separated/Divorced vs. Single & 0.71 & 2.04 & $(1.30-3.20)$ & 9.60 & 0.002 \\
\hline Widowed vs. Single & 0.75 & 2.12 & $(0.87-5.14)$ & 2.76 & 0.096 \\
\hline Living Arrangement & & & & 5.59 & 0.232 \\
\hline W/Spouse or Relative vs. Independent & -0.07 & 0.94 & $(0.56-1.55)$ & 0.07 & 0.794 \\
\hline W/Parents vs. Independent & -0.25 & 0.78 & $(0.44-1.37)$ & 0.76 & 0.384 \\
\hline W/Non-Relatives vs. Independent & 0.36 & 1.43 & $(0.79-2.56)$ & 1.40 & 0.237 \\
\hline Residential Home ${ }^{\text {a }}$ vs. Independent & -1.03 & 0.36 & $(0.10-1.34)$ & 2.33 & 0.127 \\
\hline Employment Status & & & & 11.87 & 0.018 \\
\hline Student vs. Unemployed & -1.31 & 0.27 & $(0.12-0.62)$ & 9.56 & 0.002 \\
\hline Employed vs. Unemployed & 0.09 & 1.09 & $(0.68-1.74)$ & 0.13 & 0.722 \\
\hline Disability vs. Unemployed & -0.27 & 0.77 & $(0.45-1.28)$ & 1.05 & 0.306 \\
\hline Retired vs. Unemployed & -0.25 & 0.78 & $(0.33-1.86)$ & 0.31 & 0.578 \\
\hline Referral Source & & & & 2.62 & 0.623 \\
\hline Physician/Hospital vs. Residence & -0.15 & 0.86 & $(0.48-1.55)$ & 0.26 & 0.611 \\
\hline Psychiatric Facility vs. Residence & -0.65 & 0.52 & $(0.11-2.42)$ & 0.69 & 0.406 \\
\hline Residential Home ${ }^{\mathrm{a}}$ vs. Residence & -1.11 & 0.33 & $(0.06-1.70)$ & 1.75 & 0.185 \\
\hline Other vs. Residence & 0.03 & 1.03 & $(0.65-1.64)$ & 0.02 & 0.900 \\
\hline Insurance Type & & & & 11.64 & 0.003 \\
\hline Medicaid/Medicare vs. Self-Pay & -1.01 & 0.37 & $(0.20-0.66)$ & 11.11 & 0.001 \\
\hline Private Health Plan vs. Self-Pay & -0.35 & 0.70 & $(0.44-1.12)$ & 2.17 & 0.140 \\
\hline \multicolumn{6}{|l|}{ Clinical and Medical Characteristics } \\
\hline \multicolumn{6}{|l|}{ Presenting Problem(s) } \\
\hline Violence & 0.46 & 1.58 & $(0.73-3.42)$ & 1.36 & 0.244 \\
\hline Homicidal Intent & -2.13 & 0.12 & $(0.02-0.92)$ & 4.16 & 0.041 \\
\hline \multicolumn{6}{|l|}{ Affect } \\
\hline Anxious & -0.43 & 0.65 & $(0.46-0.92)$ & 5.79 & 0.016 \\
\hline Depressed & 0.33 & 1.39 & $(0.98-1.98)$ & 3.42 & 0.064 \\
\hline \multicolumn{6}{|l|}{ Medical History } \\
\hline Diabetes & -0.45 & 0.67 & $(0.26-1.55)$ & 0.99 & 0.319 \\
\hline "Prescribed Meds & -0.03 & 0.97 & $(0.90-1.04)$ & 0.68 & 0.409 \\
\hline
\end{tabular}

a. Residential homes include nursing homes, group homes, or assisted living facilities.

who were students rather than unemployed $(O R=0.27, p=0.002)$ and for those who were on public assistance (i.e., Medicaid or Medicare) rather than self-pay clients $(O R=0.37, p<0.001)$. The odds that emergency psychiatry patients' presenting problems were substance-related were also significantly reduced among homicidal $(\mathrm{OR}=0.12, \mathrm{p}=0.041$ ) or anxious ( $\mathrm{OR}=0.65, \mathrm{p}=0.016)$ patients.

Using the same set of predictors that were entered into the first logistic regression model, a second logistic regression was run in order to predict admissions whose only presenting problem was substance involvement. The previous two predictors dealing with presenting problems (i.e., violence, homicide) were left out of the model, though, because they could not occur in cases whose only presenting problem was substance-related, which was what this model was predicting. The overall model was statistically significant, $\chi^{2}(22)=71.94, p<0.0001$, Nagelkerke
$R^{2}=0.18$. The model was similar to the previous one except that gender, employment status, and insurance type were no longer significant predictors. Separated or divorced participants were more than three times as likely $(O R=3.30, p<0.001)$ as those who were single to present for services for only substance-related reasons. The odds that emergency psychiatry clients' presenting problems were only related to substance involvement were also significantly reduced among anxious patients $(O R=0.54, p=0.031)$.

\section{Limitations}

This study did not use intake interviews that were previously validated in other investigations. Rather, interviewers conducted standardized psychiatric interviews that were developed in conjunction with their clinical and administrative staff. As such, 
this study can be considered to be naturalistic in the sense that assessment materials were more representative of what is commonly used in other emergency psychiatry departments located outside of University settings. Another limitation is the fact that interview findings regarding substance involvement were not corroborated with biological screening tests. Last, no information on the specific substance (e.g., alcohol, cocaine) and severity of problems was obtained.

\section{Discussion}

Traditional behavioral health service systems tend to focus on either mental health or substance abuse treatment services. The elevated rates of co-occurring mental health and substance use disorders consistently documented among emergency psychiatry patients underscores the insufficiency of the fragmented system in offering effective, integrated services for this population. Identifying substance misuse among emergency psychiatry patients is a necessary first step towards addressing their treatment needs. Once identified, patients can be offered a brief intervention and/or can be referred to substance abuse treatment services as appropriate. Because of the severe nature of the problems prompting patients to seek emergency psychiatry services, the emergency department visit itself has been referred to as a teachable moment in which patients may be more receptive to advice or referral for additional services. Brief substance abuse counseling interventions delivered in emergency departments $[19,20]$ have proven effective, and there is evidence that motivational interviewing can effectively be used as brief interventions in these settings [21], even when implemented by staff not specializing in addictions treatment. Barrett and colleagues (2006) used a randomized controlled trial to demonstrate the effectiveness of having an alcohol health worker (AHW) deliver a brief intervention to patients with hazardous drinking patterns [22]. Six-month follow-up results indicated that patients randomly assigned to the AHW condition drank significantly less than patients assigned to an information only control condition, and that delivering the AHW condition was no more costly than the information only control.

Obtaining information on emergency psychiatry patient substance use can also shed light on other important aspects of the emergency psychiatry treatment process, as it has been linked both to higher likelihood of leaving the hospital against medical advice and also to lower follow-through on referrals. For instance, Solomon and Gordon (1988) found that emergency psychiatry patients identified as having substance-related problems were significantly less likely than those without substance use problems to follow up on referrals made to community treatment agencies [15]. Bradley and Zarkin (1997) noted that individuals with substance use disorders were more likely to leave the hospital against medical advice [7].

Results of the present study confirm the high rates of substance use among emergency psychiatry patients found in prior studies. Importantly, this study involved administration of a standardized interview to a large sample of over 2,000 consecutive admissions to three separate emergency psychiatry centers. While the structured interviews revealed that over $23 \%$ of admissions presented for emergency psychiatry services due at least in part to their involvement with alcohol or drugs, nearly $8 \%$ presented for services due solely to their involvement with these substances. Though prior studies have documented high rates of substance involvement among emergency psychiatry patients, this study extends those findings by further examining characteristics associated with admissions whose presenting problems were noted as consisting solely of substancerelated issues. This extension is important, especially provided the well documented costly nature of these individuals' service needs.

Analyzing data from the 2004 National Hospital Ambulatory Medical Care Survey (NHAMCS), Kunen and colleagues (2006) found that individuals with psychiatric comorbidity treated in emergency rooms were five times more likely to be hospitalized than those with a single psychiatric disorder [23]. Further, they reported that the most frequent psychiatric comorbidities involved substance use disorders. Individuals with unmet substance abuse treatment needs who received services in an emergency room were $81 \%$ more likely to be admitted during their emergency room visit, and were $46 \%$ more likely to have reported making at least one ER visit in the previous 12 months [24]. These authors suggested that each ER patient with an unaddressed addiction problem cost an additional $\$ 1,568$. Therefore, aside from being able to help patients secure assistance for their substance abuse treatment needs, appropriate identification of such needs can result in significant cost savings for hospital-based emergency care settings.

Mc Alpine and Mechanic's (2002) analysis over 2,600,000 emergency room admissions by clients with a primary mental health diagnosis found that most patients (36\%) were either on public assistance (Medicare or Medicaid) or had no insurance (26\%), with fewer being covered by private insurance $(25 \%)$ or another form of insurance (14\%) [25]. Solomon and Gordon (1988) similarly noted public assistance to be the primary payer source among their sample of 114 emergency psychiatry patients [15]. Findings from our total sample of emergency psychiatry patients resemble these, but our results extend these by indicating that a subset of substance-involved emergency psychiatry patients differ significantly in their sources of insurance coverage. Our results indicate that substance-involved emergency psychiatry admissions were significantly more likely to be uninsured, whereas the cohort of emergency psychiatry admissions who's presenting problems were not documented to be substancerelated were significantly more likely to be on public assistance. Though the groups did not differ in their likelihood of being on private insurance, the finding that substance-involved emergency psychiatry admissions were significantly more likely to be uninsured is a cause of concern alongside findings indicating that this population also requires more intensive and costly services [12-14], including a greater number of emergency department readmissions if substance use remains unaddressed. Thus, improved identification, referral, and treatment of substance use disorders in emergency departments could result in decreased use of emergency healthcare services and overall costs as well as improved patient health outcomes.

Despite their increased costs, substance involved emergency psychiatry patients do not exhibit increased violence when 
presenting for services. Consistent with findings reported by McNiel and Binder (2005) and Sanguineti and Brooks (1992), this study found that substance-involved emergency psychiatry admissions were less likely to exhibit violent behavior in the emergency psychiatry room than their non-using counterparts $[26,27]$. Sanguineti and Brooks (1992) screened for substance use with a biological screening test (urine toxicology screen), while McNeil and Binder (2005) used interview methods similar to those employed in the current study to assess substance-involvement. Thus both interview- and biologically-based methods of screening for substance use have demonstrated a consistent relationship between substance involvement and reduced violence in the emergency psychiatry service setting.

Previous studies have failed to reach consensus with regard to the relationship between suicidality and substance use among emergency psychiatry patients. While Sanguineti and Brooks (1992) found that emergency psychiatry patients screening negative for substances were more likely to be committed for suicidal behavior (total sample size=247), Breslow et al. (1996) found that substance abusing psychiatric emergency room patients were more likely to present as suicidal (total sample=294). Using a markedly larger sample of emergency psychiatry patients which resulted in increased statistical power to detect significant differences, the present study of 2,161 consecutive admissions failed to find any differences in rates of suicidality between emergency psychiatry patients varying in their substance involvement. Though it may be possible that specific substances like alcohol may be related to increased suicidality among emergency psychiatry patients, these studies failed to differentiate between different drugs of abuse.

Because limited resources often preclude universal or widespread implementation of costly biologically-based alcohol and drug screening procedures in emergency psychiatry settings, the current study's authors agree with recommendation promoted by Dhossche and Rubinstein (1996) and Roche et al. (2006) that biologically-based screens be used only among those exhibiting the greatest risk factors as revealed through interview procedures [11-28]. The current study's findings indicate that risk factors for substance involved emergency psychiatry admissions include male gender, being separated or divorced versus single, being unemployed versus a student, and being self-pay versus having Medicaid/Medicare. Over and above these risk factors, however, it is imperative that emergency psychiatry staff's professional judgment always plays a central role in decisions such as whether or not to drug test a patient. Absence of risk factors should never compromise staff's attention to and care of a patient. Last, we agree with recommendations made by Touquet (2005) and Alley et al. (2006) that interview-based procedures may not be feasible to conduct with patients who are not alert and orientated. In such instances, professional judgment and standard protocols should be used $[29,30]$. 


\section{References}

1 Regier DA, Farmer ME, Rae DS (1990) Comorbidity of mental disorders with alcohol and other drug abuse. Journal of the American Medical Association 264: 2511-2518.

2 Regier DA, Narrow WE, Rae DS, Manderscheid RW, Locke BZ, et al. (1993) The defacto U.S. mental and addictive disorders service system: Epidemiological Catchment Area prospective 1-year prevalence rates of disorders and services. Archives of General Psychiatry 50: 85-94.

3 Kessler RC, McGonagle KA, Zhao S, Nelson CB, Hughes M, et al. (1994) Lifetime and 12-month prevalence of DSM-II-R psychiatric disorders in the United States: Results from the National Co-Morbidity Survey. Archives of General Psychiatry 51: 8-19.

4 Binks S, Hoskins R, Salmon, Benger J (2005) Prevalence and healthcare burden of illicit drug use among emergency department patients. Emergency Medicine Journal 22: 872-873.

5 Cherpitel CJ (1999) Emergency room and primary care service utilization and associated alcohol and drug use in the United States general population. Alcohol and Alcoholism, 34: 581-589.

6 Larson M, Saitz R, Horton N, Lloyd-Travaglini C, Samet J (2006) Emergency department and hospital utilization among alcohol and drug-dependent detoxification patients without primary medical care. The American Journal of Drug and Alcohol Abuse 32: 435-452.

7 Bradley CJ, Zarkin GA (1997) An inpatient profile of patients with a substance abuse diagnosis in Maryland. Journal of Substance Abuse Treatment 14: 155-162.

8 Fong T, Tsuang J, Ho A, Haponski D, Giannini C (2002) Factors related to poor contact of discharged dual diagnosis patients from a psychiatric emergency room. Addictive Disorders and Their Treatment 1: 81-87.

9 Padgett DK, Struening EL (1991) Influence of substance abuse and mental disorders on emergency room use by homeless adults. Hospital and Community Psychiatry 42: 834-838.

10 Schiller MJ, Shumway M, Balki SL (2000) Patterns of substance use among patients in an urban psychiatric emergency service. Psychiatric Services 51: 113-115.

11 Roche AM, Freeman T, Skinner N (2006) From data to action: Findings from a systematic review of hospital screening studies for high-risk alcohol consumption. Drug and Alcohol Dependence 83: 1-14.

12 Breslow RE, Klinger BI, Erickson AJ (1996) Acute intoxication and substance abuse among patients presenting to a psychiatric emergency service. General Hospital Psychiatry 18: 183-191.

13 Curran GM, Sullivan G, Williams K, Han X, Collins K, et al. (2003) Emergency department use of persons with comorbid psychiatric and substance abuse disorders. Annals of Emergency Medicine 41: 659-667.

14 Wildt BT, Andreis C, Auffahrt I, Tettenborn C, Kropp S, et al. (2006) Alcohol related conditions represent a major psychiatric problem in emergency departments. Emergency Medicine Journal 23: 428-430.

15 Solomon P, Gordon B (1988) Follow-up outpatient referrals from a psychiatric emergency room. Social Work in Health Care 13: 57-67.

16 Baker Act Handbook (The Florida Mental Health Act): User Reference Guide. (2004). Mental Health Program Office, Florida Department of Children and Families, State of Florida.

17 Florida Statutes (2004), section 394.

18 Kirk RE. (1995) Experimental design: Procedures for the behavioral sciences. Pacific Grove, CA: Brooks/Cole Publishing Company.
19 Crawford MJ, Patton R, Touquet R, Drummond C, Byford S, et al (2004) Screening and referral for brief intervention of alcohol misusing patients in an emergency department: A pragmatic randomized controlled trial. Lancet 364: 1334-1339.

20 Longabagaugh R, Woolard RF, Nirenberg TD, Minugh AP, Becker B, et al. (2001) Evaluating the effects of a brief motivational intervention for injured drinkers in the emergency department. Journal of Studies on Alcohol 62: 806-816.

21 Dunn C, Deroo L, Rivara FP (2001) The use of brief interventions adapted from motivational interviewing across behavioral domains: A systematic review. Addiction 96: 1725-1742.

22 Barrett B, Byford S, Crawford M, Patton R, Drummond C, et al. (2006) Cost-effectiveness of screening and referral to an alcohol health worker in alcohol misusing patients attending an accident and emergency department: A decision-making approach. Drug and Alcohol Dependence 81: 47-54.

23 Kunen S, Prejean C, Gladney B, Harper D, Mendry CV (2006). Disposition of emergency department patients with psychiatric comorbidity: Results from the 2004 National Hospital Ambulatory Medical Care Survey. Emergency Medicine Journal 23: 274-275.

24 Rockett IRH, Putnam SL, Jia H, Chang CF, Smith GS (2005) Unmet substance abuse treatment need, health services utilization, and cost: A population-based emergency department study. Annals of Emergency Medicine 45: 118-127.

25 McAlpine DD, Mechanic D (2002) Data points: Payer sources for emergency room visits by persons with psychiatric disorders. Psychiatric Services 53: 14.

26 McNiel DE, Binder RL (2005) Psychiatric emergency service use and homelessness, mental disorder, and violence. Psychiatric Services 56: 699-704.

27 Sanguineti V, Brooks M (1992) Factors related to emergency commitment of chronic mentally ill patients who are substance abusers. Hospital and Community Psychiatry 43: 237-241.

28 Dhossche D, Rubinstein J (1996) Drug detection in a suburban psychiatric emergency room. Annals of Clinical Psychiatry 8: 59-69.

29 Touquet R (2005) Clinical assessment compared with breathalyzer readings in the ER: concordance of ICD-10 Y90 and Y91 codes. Emergency Medicine Journal 22: 695.

30 Alley P, Graham W, McDonnell M, Spedding R (2006) Alcohol levels in the emergency department: A worrying trend. Emergency Medicine Journal 23: 707-708.

31 Atkinson R (1973) The importance of alcohol and drug abuse in psychiatric emergencies. California Medical Journal 118: 1-4.

32 Claassen CA, Gilfillan S, Orsulak P, Carmody TJ, Battaglia J, et al. (1997) Substance use among patients with a psychotic disorder in a psychiatric emergency room. Psychiatric Services, 48: 353-358.

33 Elangovan N, Berman S, Meizner A, Gianelli P, Miller H, et al. (1993) Substance Abuse among patients presenting at an inner-city psychiatric emergency room. Hospital and Community Psychiatry 44: 782-784.

34 Gilfillan S, Claassen CA, Orsulak P, Carmoody TJ, Sweeney JB, et al. (1998) A comparison of psychotic and nonpsychotic substance users in the psychiatric emergency room. Psychiatric Services 49: 825-828.

35 Lejoyeux $M$, Boulenguiez $S$, Fichelle $A$, McLoughlin $M$, Claudon $M$, et al. (2000) Alcohol dependence among patients admitted to psychiatric emergency services. General Hospital Psychiatry 22: 206-212.

36 Lieberman $P$ and Baker $F(1985)$ The reliability of psychiatric diagnosis in the emergency room. Hospital and Community Psychiatry 36: 291-293. 
37 Strakowski S, Loczzak H, Sax K, West S, Crist A, et al. (1995) The effects of race on diagnosis and disposition from a psychiatric emergency service. Journal of Clinical Psychiatry 56: 101-107.

38 Szuster RR, Schanbacher BL, McCann SC (1990) Characteristics of psychiatric emergency room patients with alcohol- or drug-induced disorders. Hospital and Community Psychiatry 41: 1342-1345.

39 Sanguineti VR, Samuel SE (1993) Reported prevalence of drug abuse comorbidity in a city-wide emergency rooms system. American Journal of Drug and Alcohol Abuse, 19: 443-450. 